\title{
‘Intention-Behaviour Gap’
}

David F Marks

Potential competing interests: The author(s) declared that no potential competing interests exist.

The 'intention-behaviour gap' occurs when a person develops an intention to change their behaviour (e.g., to wear a face mask), but they do not take any action (e.g., actually to wear a face mask). This discrepancy has been labelled the 'intention-behaviour gap'. 